\title{
Influência do modo de armazenamento na microinfiltração de dentes decíduos restaurados com diferentes sistemas adesivos: estudo in vitro
}

\author{
Influence of the method of storage on the microleakage \\ of primary teeth restored with different bonding \\ systems: in vitro study
}

Eloisa Lorenzo de Azevedo GHERSEL*

Antônio Carlos GUEDES-PINTO**

Ana Lídia CIAMPONI***

\begin{abstract}
GHERSEL, E. L. de A.; GUEDES-PINTO, A. C.; CIAMPONI, A. L. Influência do modo de armazenamento na microinfiltração de dentes decíduos restaurados com diferentes sistemas adesivos: estudo in vitro. Pesqui Odontol Bras, v. 15, n. 1, p. 29-34, jan./mar. 2001.
\end{abstract}

\begin{abstract}
Foi avaliada, in vitro, a influência do modo de armazenamento e de dois tipos de adesivos dentinários sobre a microinfiltração nas paredes axiais e cervicais de dentes decíduos restaurados com resina composta. As amostras foram divididas de acordo com o modo com que foram armazenadas e os grupos foram classificados em: Congelado, Hidratado e Desidratado. O grupo Congelado foi mantido no "freezer", em solução fisiológica, o grupo Hidratado foi armazenado em solução fisiológica em geladeira e o grupo Desidratado foi mantido seco. Foram realizados preparos tipo "slots" verticais: ocluso-mesial e ocluso-distal. Nos preparos ocluso-mesiais, utilizou-se o adesivo Scotchbond Multi-Uso e nos ocluso-distais o Prime \& Bond 2.1, sendo todos restaurados com resina composta Solitaire. Após termociclagem, foram imersos em corante e os valores de microinfiltração mensurados através de sistema de imagens digitalizadas e submetidos à análise de variância. Os resultados demonstraram que o modo de armazenamento não apresentou influência estatisticamente significante na microinfiltração das restaurações. A microinfiltração na parede cervical foi significantemente maior que na parede axial, com segurança de 99,9\%. Os adesivos utilizados não apresentaram influência significante na microinfiltração, nas diferentes formas de armazenamento estudadas. Porém, houve diferença estatisticamente significante nas amostras do grupo Desidratado, com o adesivo Prime $\&$ Bond 2.1, considerando as margens da restauração (axial e cervical).
\end{abstract}

UNITERMOS: Dente decíduo; Infiltração dentária.

\section{INTRODUÇÃO}

Com o aprimoramento dos trabalhos laboratoriais que visam simular condições bucais, inúmeras pesquisas têm sido desenvolvidas utilizando-se dentes naturais em testes com materiais odontológicos. No caso específico da Odontopediatria, os dentes decíduos esfoliados têm sido utilizados tanto para testes de laboratório como para trabalhos clínicos em restaurações biológicas.

As pesquisas realizadas para avaliação da microinfiltração in vitro tentam simular as condições in vivo, não havendo todavia metodologia padronizada, totalmente definida, em relação a armazenagem $^{1,2,5,10,15,23}$.
Sempre que se trabalha com dentes extraídos, é importante que estes sejam submetidos a métodos de desinfecção ou esterilização como autoclave, que é um método seguro ${ }^{19}$. Já para o armazenamento, diversas soluções são empregadas, e a mais comumente usada é a solução fisiológica, acrescida de agentes antibacterianos ${ }^{8}$ ou na forma pura $^{1,8}$.

Apesar de o tempo de armazenamento ser considerado uma variável importante por muitos autores, tendo apresentado influência significante nos estudos de STRAWAN et al. ${ }^{22}$ (1996) e GOODIS et al. ${ }^{13}$ (1991), não demonstrou significância na maioria dos trabalhos pesquisados ${ }^{10,17,18}$, não tendo

* Professora da Disciplina de Odontopediatria da UFMS.

** Professor Titular; *** Professora - Disciplina de Odontopediatria da Faculdade de Odontologia da USP. 
GHERSEL, E. L. de A.; GUEDES-PINTO, A. C.; CIAMPONI, A. L. Influência do modo de armazenamento na microinfiltração de dentes decíduos restaurados com diferentes sistemas adesivos: estudo in vitro. Pesqui Odontol Bras, v. 15, n. 1, p. 29-34, jan./mar. 2001.

sido portanto objetivo do nosso estudo avaliar essa variável.

O meio de armazenamento de dentes extraídos, entretanto, pode provocar alterações químicas e ópticas na superfície dentinária ${ }^{21}$, afetar a resistência adesiva ${ }^{8}$, influenciar a permeabilidade da dentina $^{13}$ e influenciar a própria microinfiltração $0^{1,2,15}$.

Para outros autores, a forma de armazenamento de dentes não afetou a resistência adesiva ${ }^{17}$, nem a permeabilidade dentinária ${ }^{6,18}$.

Diversos estudos têm sido realizados avaliando-se a eficiência dos sistemas adesivos associados a resinas compostas ${ }^{11,20}$, destacando-se a qualidade da camada híbrida ${ }^{3}$. Embora muito se tenha conseguido em termos de eficiência, os problemas relativos à microinfiltração ainda não foram totalmente superados, principalmente nas margens cervicais $^{12,22}$.

Diante do exposto, este estudo propõe-se a avaliar, in vitro, a influência do modo de armazenamento e de dois tipos de adesivos dentinários sobre a microinfiltração marginal nas paredes axiais e cervicais de molares decíduos, quando restaurados com resina composta.

\section{MATERIAL E MÉTODOS}

Foram selecionados vinte e quatro segundos molares decíduos, hígidos, extraídos em época de esfoliação, que foram divididos aleatoriamente em três grupos de oito e classificados de acordo com o modo de armazenamento em: Congelado, Hidratado e Desidratado.

Os dentes foram lavados em água corrente, com escova de "nylon", para remoção dos tecidos aderidos. Logo após, foram esterilizados em autoclave ${ }^{19}$ por 20 minutos, a $121^{\circ} \mathrm{C}$. Aqueles pertencentes ao grupo Congelado foram imersos em soro fisiológico a $0,9 \%$, sem o uso de qualquer solução antibacteriana, num frasco plástico tampado e colocados no "freezer" no máximo por 30 dias, tendo sido colocados em refrigerador 24 horas antes do início do trabalho, para descongelamento. Os dentes pertencentes ao grupo Hidratado foram colocados na mesma solução, em recipiente de vidro tampado e mantidos no refrigerador ${ }^{1}$, no máximo por 30 dias, antes do trabalho de laboratório, sendo essa solução trocada a cada vinte e quatro horas. Os dentes do grupo Desidratado foram mantidos secos, em recipientes plásticos abertos, à temperatura ambiente, no máximo por 30 dias antes do início do trabalho. Uma hora antes de receber o preparo cavitário, foram hidratados em água destilada.
Foram preparadas cavidades de classe II, do tipo "slot" vertical, ocluso-mesial e ocluso-distal, em alta rotação, com brocas "carbide" número 245 , trocadas a cada três preparos, para que fosse possivel manter a uniformidade em todas elas. As medidas dos preparos ficaram em torno de $3 \mathrm{~mm}$ de largura, por $3 \mathrm{~mm}$ de altura e $2 \mathrm{~mm}$ de profundidade, conferidas com um compasso de ponta seca, com o objetivo de preservar as devidas proporções, de acordo com a metodologia de MIRANDA Jr. ${ }^{17}$ (1992); TERUYA $^{23}$ (1994); $\operatorname{IMPARATO}^{16}(1996)$ e BUSSADORI ${ }^{4}$ (1997). Os ângulos internos do preparo foram arredondados e os ângulos cavossuperficiais ficaram localizados em esmalte, inclusive os da parede cervical. Não foi realizado o bisel cavossuperficial em esmalte. Todas as cavidades foram restauradas com resina composta Solitaire, porém nas cavidades oclusomesiais, foi utilizado o adesivo Scotchbond Multi-Uso e, nas cavidades ocluso-distais, o adesivo Prime \& Bond 2.1, de acordo com as recomendações do fabricante. Após acabamento e polimento, as amostras foram imersas em água destilada por 24 horas antes de serem submetidas à termociclagem, passando por 700 ciclos térmicos, com 30 segundos em cada banho, nas temperaturas de $5^{\circ} \mathrm{C}$ a $55^{\circ} \mathrm{C}$, com intervalo de transferência de 6 segundos entre os banhos ${ }^{7,10}$.

As raizes remanescentes foram removidas com disco de carborundum e a base do dente foi recoberta com resina acrílica autopolimerizável, do tipo Ortoclas. A seguir, foram aplicadas duas camadas de esmalte cosmético, vermelho, pinceladas em toda a superficie dos dentes, exceto em uma faixa, localizada na face proximal, incluindo toda a restauração e ultrapassando em $2 \mathrm{~mm}$ a interface do dente e do material restaurador, para permitir o contato com o corante exclusivamente através dessa região.

Após impermeabilização, as amostras foram imersas em solução de nitrato de prata a $50 \%{ }^{5}$, pH 6,7 e deixadas no escuro por duas horas. Logo após, foram lavadas em água corrente por um minuto e colocadas em água destilada, por mais um minuto, para remover as partículas de prata aderidas à superficie. Em seguida, foram mergulhadas no revelador fotográfico (Kodak), por um período de oito horas, sob luz fluorescente, com finalidade de precipitar as partículas de prata. Findo esse período, os dentes foram lavados com água destilada para remover o revelador e estocados em água destilada até serem cortados para posterior leitura ${ }^{23}$.

As amostras, num total de 24 , foram primeira- 
GHERSEL, E. L. de A.; GUEDES-PINTO, A. C.; CIAMPONI, A. L. Influência do modo de armazenamento na microinfiltração de dentes decíduos restaurados com diferentes sistemas adesivos: estudo in vitro. Pesqui Odontol Bras, v. 15, n. 1, p. 29-34, jan./mar. 2001.

mente seccionadas no sentido vestíbulo-lingual, com disco de carborundum, resultando em 48 partes e incluídas em resina acrílica autopolimerizável, assim como suas respectivas identificações para receber um segundo corte agora no sentido mésio-distal, seccionando-se o centro da restauração com disco diamantado, obtendo-se 96 espécimes.

Os valores de microinfiltração foram mensurados através de sistema de imagens digitalizadas e foram avaliadas duas posições, a interface dente-restauração da parede axial e da parede cervical, perfazendo o total de 192 medidas submetidas à análise de variância.

\section{RESULTADOS}

Os dados iniciais foram computados com precisão de centésimos de milimetros, através de medições com o software Diracon III. A seguir, foram submetidos a análise de variância, utilizando-se o recurso "split-plot" e os resultados encontram-se dispostos na Tabela 1. Em relação ao modo de armazenamento, não houve diferença estatisticamente significante na microinfiltração das amostras, independentemente do material e da interface dente-restauração das paredes axiais e cervicais. Houve, porém, diferença estatisticamente significante, com segurança de 99,9\% quando comparadas as medições na interface axial e cervi-

TABELA 1 - Análise de variância dos valores de microinfiltração correspondentes aos fatores: armazenamento, adesivo e interface, e suas interações.

\begin{tabular}{l|r|c|c|c}
\hline \hline Fonte de variação & G.L. & $\begin{array}{c}\text { Soma dos } \\
\text { quadrados }\end{array}$ & $\begin{array}{c}\text { Quadrados } \\
\text { médios }\end{array}$ & $(\mathrm{F})$ \\
\hline Armazenamento (A) & 2 & 0,1830 & 0,0915 & $0,30 \mathrm{~ns}$ \\
\hline Material (M) & 1 & 0,6924 & 0,6924 & $2,28 \mathrm{~ns}$ \\
\hline Interação (A x M) & 2 & 0,3323 & 0,1662 & $0,55 \mathrm{~ns}$ \\
\hline Resíduo I & 90 & 27,3118 & 0,3035 & - \\
\hline Interface (I) & 1 & 2,1611 & 2,1611 & $15,85^{* *}$ \\
\hline Interação (I x A) & 2 & 0,4249 & 0,2124 & $1,56 \mathrm{~ns}$ \\
\hline Interação (I x M) & 1 & 0,0142 & 0,0142 & $0,10 \mathrm{~ns}$ \\
\hline Interação \\
(I x A x M) & 2 & 1,1363 & 0,5681 & $4,17 *$ \\
\hline Resíduo II & 90 & 12,2727 & 0,1364 & - \\
\hline Total & 191 & 44,5287 & - & - \\
\hline \hline
\end{tabular}

ns - não significante; * significante com segurança de 95\%; ** significante com segurança de 99,9\%. cal. Foram também estatisticamente significantes, com segurança de $95 \%$, as interações dos fatores armazenamento, material e interface.

Na Tabela 2, encontram-se as médias em milímetros, correspondentes aos fatores armazenamento, material e interface. As médias resultantes do modo de armazenamento não apresentaram diferenças estatisticamente significantes. Mesmo não apresentando diferenças estatisticamente significantes, as médias para os tipos de adesivo mostram diferença numérica sugestiva, comparando-se o Scotchbond Multi-Uso $(0,41 \mathrm{~mm})$ e o Prime \& Bond 2.1 (0,29 mm), com maior microinfiltração para o primeiro. Houve, porém, diferenças significantes com segurança de $99,9 \%$ para o fator interface, quando comparadas as médias das interfaces axial $(0,24 \mathrm{~mm})$ com a cervical $(0,46 \mathrm{~mm})$, demonstrando maior infiltração nesta última.

Os valores das médias de microinfiltração dos fatores armazenamento $\mathrm{x}$ material $\mathrm{x}$ interface interagem-se na Tabela 3. Demonstram que não houve diferenças estatisticamente significantes, exceto para os valores entre as amostras do grupo Desidratado, quando restauradas com o adesivo Prime $\&$ Bond 2.1, nas interfaces dente-restauração das paredes axiais e cervicais.

\section{DISCUSSÃO}

Os resultados demonstraram que não houve diferença estatisticamente significante na microinfiltração, em relação ao modo de armazenamento, mesmo quando se variou o tipo de material, como demonstra a Tabela 1. Esse fato pode ter ocorrido pela associação de fatores, como a padronização da profundidade do preparo (aproximadamente

TABELA 2 - Médias de microinfiltração em milímetros correspondentes aos fatores armazenamento, material e interface.

\begin{tabular}{c|c|c|c}
\hline \hline \multicolumn{3}{c|}{ Armazenamento } & \multirow{2}{*}{$\begin{array}{c}\text { Significância } \\
(\%)\end{array}$} \\
\hline Congelado & Hidratado & Desidratado & \\
\hline 0,32 & 0,35 & 0,39 & \multirow{2}{*}{$\begin{array}{c}\text { não } \\
\text { significante }\end{array}$} \\
\hline $\begin{array}{c}\text { Scotchbond } \\
\text { Multi-Uso }\end{array}$ & Prime \& Bond 2.1 & \\
\hline 0,41 & 0,29 & \multirow{2}{*}{0,1} \\
\hline \multicolumn{3}{c|}{ Interface } \\
\hline Axial & Cervical & \\
\hline 0,24 & 0,46 & \\
\hline \hline
\end{tabular}


GHERSEL, E. L. de A.; GUEDES-PINTO, A. C.; CIAMPONI, A. L. Influência do modo de armazenamento na microinfiltração de dentes decíduos restaurados com diferentes sistemas adesivos: estudo in vitro. Pesqui Odontol Bras, v. 15, n. 1, p. 29-34, jan./mar. 2001.

TABELA 3 - Médias de microinfiltração (em milimetros) correspondentes à interação armazenamento $\mathrm{x}$ material $\mathrm{x}$ interface e valor crítico para contraste.

\begin{tabular}{|c|c|c|c|c|c|}
\hline \multirow[b]{2}{*}{ Material } & \multirow[b]{2}{*}{ Interface } & \multicolumn{3}{|c|}{ Armazenamento } & \multirow{2}{*}{$\begin{array}{c}\text { Tukey } \\
(5 \%)\end{array}$} \\
\hline & & $\begin{array}{c}\text { Conge- } \\
\text { lado }\end{array}$ & $\begin{array}{l}\text { Hidra- } \\
\text { tado }\end{array}$ & $\begin{array}{l}\text { Desidra- } \\
\text { tado }\end{array}$ & \\
\hline \multirow{2}{*}{$\begin{array}{l}\text { Scotchbond } \\
\text { Multi-Uso }\end{array}$} & Axial & 0,33 & 0,22 & 0,38 & \multirow{4}{*}{0,438} \\
\hline & Cervical & 0,52 & 0,50 & 0,51 & \\
\hline \multirow{2}{*}{$\begin{array}{l}\text { Prime \& } \\
\text { Bond } 2.1\end{array}$} & Axial & 0,10 & 0,37 & 0,05 & \\
\hline & Cervical & 0,30 & 0,31 & 0,60 & \\
\hline
\end{tabular}

$2 \mathrm{~mm}$, sendo $1 \mathrm{~mm}$ em esmalte e $1 \mathrm{~mm}$ em dentina), confirmando o estudo de MITCHEM; GRONAS $^{18}$ (1986) em que, além de o modo de armazenamento não ter influenciado significantemente os resultados, a maior resistência adesiva foi conseguida à distância de $2 \mathrm{~mm}$ da polpa. Da mesma forma, a pesquisa de OUTHWAITE et al. ${ }^{19}$ (1996) demonstrou que quanto menos se aprofunda na dentina, menor é a permeabilidade dentinária e, conseqüentemente, melhor a adesão. Outro fator que pode ter contribuído para a uniformidade dos resultados pode ter sido a imersão simultânea de todas as amostras em água destilada por um período 24 horas, após o acabamento e polimento da restauração, o que promove absorção de água pela resina composta, expandindo a restauração e melhorando o selamento marginal, corroborando com os estudos de CRIM; GARCIA-GODOY ${ }^{10}$ em 1987. Além disso, como apenas um tipo de resina composta foi utilizado, toda a alteração dimensional sofrida pelo material foi uniforme e, mesmo os adesivos sendo de marcas comerciais diferentes, as alterações referentes a eles foram mínimas, uma vez que têm eficiência comprovada pela maioria das pesquisas. Nos estudos de COOLEY; DODGE ${ }^{9}$ (1989), o período e o meio de armazenamento não influenciaram significantemente os resultados quando três sistemas adesivos foram testados. Portanto, para esses autores, parece que o prazo de validade e a estabilidade dos materiais são mais importantes do que o meio ou o período de armazenamento.

Embora as médias de microinfiltração correspondentes ao modo de armazenamento e material não tenham apresentado diferenças significantes (Tabela 2), os valores numéricos sugerem que as amostras do grupo Desidratado permitiram maior infiltração que as do grupo Hidratado, e que as amostras do grupo Congelado apresentaram de- sempenho melhor do que os outros dois grupos. Resultados semelhantes também foram obtidos por ARAÚJO et al. ${ }^{1}$ (1998), com o maior grau de infiltração para os dentes desidratados e o menor para os dentes congelados, confirmando os resultados obtidos por CAMPS et $a .^{5}$ (1996) e BRÄNNSTRÖN et $a l^{2}{ }^{2}$ (1992), que também encontraram microinfiltração significantemente menor em dentes congelados. Isso pode ser atribuído ao fato de que os adesivos reagem também com a parte orgânica da dentina e talvez essa parte orgânica possa ser conservada em melhores condições por meio do congelamento. Além disso, as amostras do grupo Hidratado foram armazenadas em soro fisiológico, pois apesar de ser essa solução muito utilizada em pesquisas de materiais odontológicos, seu emprego tem sofrido algumas restrições, pela interferência sobre a permeabilidade dentinária ${ }^{13}$.

A Tabela 2 mostra também que, mesmo não apresentando diferença estatisticamente significante, as médias relativas ao material apresentam diferença numérica sugestiva de maior microinfiltração para o Scotchbond Multi-Uso $(0,41 \mathrm{~mm})$ em comparação ao Prime \& Bond 2.1 (0,29 mm). Talvez esse resultado confirme o raciocínio de BURROW et al. ${ }^{3}$ (1994), que destacam a importância da qualidade da camada híbrida na resistência adesiva. Aliado a esse fato, o sistema monocomponente Prime \& Bond 2.1 demonstrou bons resultados nos estudos de FINGER; FRITZ ${ }^{11}$ (1996); SETTEMBRINI et al. ${ }^{21}$ (1997). As médias correspondentes à microinfiltração nas interfaces das paredes axiais e cervicais apresentaram diferenças significantes, com segurança de 99,9\% (Tabela 2). Esses resultados foram ratificados por FUTATSUKI; NAKATA ${ }^{12}$ (1994) quando demonstraram que a microinfiltração não é uniforme nas margens das restaurações e que a maior infiltração ocorre nas margens proximais, provavelmente pela menor espessura do esmalte nessa área da cavidade.

Os valores das médias de microinfiltração da interação dos fatores (armazenamento x material $\mathrm{x}$ interface), representados na Tabela 3, mostram que não houve diferença estatisticamente significante, exceto para os valores das amostras do grupo Desidratado, com o adesivo Prime $\&$ Bond 2.1, quando se consideram as interfaces das paredes axiais e cervicais. Pode-se observar que houve um comportamento heterogêneo para as médias relativas a esse grupo, com o mesmo adesivo nas paredes cervicais e axiais. O menor valor numérico para as médias de microinfiltração foi para as 
GHERSEL, E. L. de A.; GUEDES-PINTO, A. C.; CIAMPONI, A. L. Influência do modo de armazenamento na microinfiltração de dentes decíduos restaurados com diferentes sistemas adesivos: estudo in vitro. Pesqui Odontol Bras, v. 15, n. 1, p. 29-34, jan./mar. 2001.

amostras do grupo Desidratado, com o adesivo Prime $\&$ Bond 2.1, nas interfaces das paredes axiais das restaurações e o maior valor numérico de microinfiltração foi para as médias das amostras do mesmo grupo, com o mesmo material, porém nas margens cervicais, razão para a diferença ser significante. Em nossa opinião, isso pode ter ocorrido por alterações na permeabilidade dentinária, talvez em decorrência do pouco tempo de hidratação das amostras (1 hora), já que nos estudos de ARAÚJO et al. ${ }^{1}$ (1998) os dentes foram reidratados por uma semana. Pode também haver associação com problemas existentes no esmalte das margens cervicais $^{12,22}$, porém, essas observações necessitam de maiores estudos para sua confirmação.

Dessa forma, apesar de muitas pesquisas demonstrarem a influência do modo de armazenamento em seus resultados ${ }^{1,2,13,15,21}$, neste estudo, tal influência não foi significante, corroborando com os resultados de outros autores ${ }^{3,6,10,17,18}$. Acreditamos que os fatores inerentes aos materiais utiliza- dos e a tentativa de padronização na metodologia do experimento podem ter sido mais relevantes do que a forma em que os dentes foram conservados. Mais estudos, porém, são necessários para a confirmação real de tais resultados.

\section{CONCLUSÕES}

Diante dos resultados obtidos, é possivel afirmar que:

- os modos de armazenamento Congelado, Hidratado e Desidratado não apresentaram diferenças estatisticamente significantes entre si;

- a microinfiltração na parede cervical foi estatisticamente maior que na parede axial;

- os tipos de adesivos não mostraram diferenças estatisticamente significantes nos três modos de armazenamento; houve porém diferenças estatisticamente significantes apenas nas amostras do grupo Desidratado, com o adesivo Prime \& Bond 2.1, quando consideradas as margens, com maior infiltração na cervical.

\footnotetext{
GHERSEL, E. L. de A.; GUEDES-PINTO, A. C.; CIAMPONI, A. L. Influence of the method of storage on the microleakage of primary teeth restored with different bonding systems: in vitro study. Pesqui Odontol Bras, v. 15, n. 1, p. 29-34, jan./mar. 2001.
}

\begin{abstract}
The influence of the method of storage of teeth and the effect of two kinds of dentin-bonding agents on the microleakage at the axial and cervical walls of composite restorations, in primary second molars, were evaluated. The samples were divided in three groups: Dehydrated, Hydrated and Frozen. The Dehydrated group was kept dry, the Hydrated group was stored in physiological saline solution under refrigeration, and the Frozen group was kept in the freezer, also immersed in physiological solution. The samples received two vertical slot preparations: a mesio-occlusal and a disto-occlusal one. In the mesio-occlusal cavities, Scotchbond Multi-Use adhesive system was used, whereas in the disto-occlusal cavities Prime \& Bond 2.1 system was used, and all cavities were filled with Solitaire composite resin. The groups were then thermocycled and immersed in silver nitrate solution. Microleakage was measured by means of a digitized image system and the values were submitted to statistical analysis. The results showed that the method of storage had no statistically significant influence on marginal microleakage. Microleakage at the cervical wall was significantly greater than that at the axial wall, with $99,9 \%$ of certainty. The adhesive systems did not show significant influence on microleakage, in the studied methods of storage. However, there was statistical difference in the Dehydrated group samples, with the use of Prime $\&$ Bond 2.1 adhesive, when considering the margins of the restoration (axial and cervical).
\end{abstract}

UNITERMS: Tooth, deciduous; Dental leakage.

\section{REFERÊNCIAS BIBLIOGRÁFICAS}

1. ARAÚJO, R. M.; ARAÚJO, M. A. M.; SilVA, R. C. S. P. et al. Influência de diferentes meios de armazenamento de dentes extraídos na infiltração marginal. In: REUNIÃO ANUAL DA SOCIEDADE BRASILEIRA DE PESQUISA ODONTOLÓGICA, 1998, Águas de São Pedro. Anais da Sociedade Brasileira de Odontologia, Águas de São Pedro : SBPqO, 1998. p. 14. [Resumo n. A025].

2. BRÄNNSTRÖM, M.; COLI, P.; BLIXT, M. Effect of tooth storage and cavity cleansing on cervical gap formation in class II glass-ionomer/composite restorations. Dent Mater, v. 8, n. 5, p. 327-331, Sept. 1992.

3. BURROW, M. F.; TAKAKURA, H.; NAKAJIMA, M. et al. The influence of age and depth of dentin on bonding. Dent Mater, v. 10, n. 4, p. 241-246, July 1994

4. BUSSADORI, S. K. Microinfiltração em dentes deciduos em função de materiais e condicionamento ácido. São Paulo, 1997. 73 p. Tese (Mestrado em Materiais Dentários) - Faculdade de Odontologia, Universidade de São Paulo.

5. CAMPS, J.; BAUNDRY, X.; BORDES, V. et al. Influence of 
GHERSEL, E. L. de A.; GUEDES-PINTO, A. C.; CIAMPONI, A. L. Influência do modo de armazenamento na microinfiltração de dentes decíduos restaurados com diferentes sistemas adesivos: estudo in vitro. Pesqui Odontol Bras, v. 15, n. 1, p. 29-34, jan./mar. 2001.

tooth cryopreservation and storage time on microleakage. Dent Mater, v. 12, n. 2, p. 121-126, Mar. 1996.

6. CAMPS, J.; MARTIN, P.; LADEQUE, P. et al. Influence of tooth cryopreservation on human dentin permeability, in vitro. Dent Mater, v. 10, n. 3, p. 210-214, May 1994.

7. CARDOSO, P. E. C.; PLÁCIDO, E. Microinfiltração de restaurações de classe $\mathrm{V}$ em resina composta usando oito novos adesivos hidrofilicos. In: REUNIÃO ANUAL DA SOCIEDADE BRASILEIRA DE PESQUISA ODONTOLÓGICA, 1998, Águas de São Pedro. Anais..., Águas de São Pedro : SBPqO, 1998. p. 18. [Resumo n. A037].

8. CAUSTON, B. E.; JOHNSON, N. W. Changes in the dentine of human teeth following extraction and their implication for in vitro studies of adhesion to tooth substance. Arch Oral Biol, v. 24, n. 3, p. 229-232, 1979.

9. COOLEY, R. L.; DODGE, W. W. Bond strength of three dentinal adhesives on recently extracted versus aged teeth. Quintessence Int, v. 20, n. 7, p. 513-516, 1989.

10. CRIM, G. A.; GARCIA-GODOY, F. Microleakage: the effect of storage and cycling duration. J Prosthet Dent, v. 57, n. 5, p. 574-576, May 1987.

11. FINGER, W. J.; FRITZ, U. Laboratory evaluation of one-component enamel/dentin bonding agents. Am J Dent, v. 9, n. 5, p. 206-210, Oct. 1996.

12. FUTATSUKI, M.; NAKATA, M. In vitro marginal leakage of class II composite resin restorations by thermal cycling. J Clin Pediatr Dent, v. 18, n. 3, p. 191-196, 1994.

13. GOODIS, H. E.; MARSHALL Jr., G. W.; WHITE, J. M. The effects of storage after extraction of the teeth on human dentine permeability in vitro. Arch Oral Biol, v. 36, n. 8, p. 561-566, 1991.

14. GROTH, E. D. B.; SANTOS, F. A. M.; KURAMOTO Jr., M.; MATSON, E. Avaliação dos efeitos da esterilização nas propriedades adesivas da dentina. In: REUNIÃO ANUAL DA SOCIEDADE BRASILEIRA DE PESQUISA ODONTOLÓGICA, 1998, Águas de São Pedro. Anais da Sociedade Brasileira de Odontologia. Águas de São Pedro : SBPqO, 1998. p. 18. [Resumo n. A039].

15. HALLER, B.; HOFMANN, N.; KLAIBER, B.; BLOCHING, U. Effect of storage media on microleakage of five dentin bonding agents. Dent Mater, v. 9, n. 3, p. 191-197, May 1993.
16. IMPARATO, J. C. P. Avaliação da microinfiltração de quatro técnicas restauradoras com resina composta nas caixas proximais em cavidades de classe II de molares deciduos. São Paulo, 1996. 93 p. Dissertação (Mestrado em Odontopediatria) - Faculdade de Odontologia, Universidade de São Paulo.

17. MIRANDA Jr., W. G. Avaliação da infiltração in vitro em caixas proximais restauradas com resinas compostas e cimento de ionômero de vidro. São Paulo, 1992. 82 p. Tese (Mestrado em Dentística Restauradora) Faculdade de Odontologia, Universidade de São Paulo.

18. MITCHEM, J. C.; GRONAS, D. G. Effects of time after extraction and depth of dentin on resin dentin adhesives. J Am Dent Assoc, v. 113, p. 285-287, 1986.

19. OUTHWAITE, W. C.; LIVINGSTON, M. J.; PASHLEY, D. H. Effects of changes in surface area, thickness, temperature and post-extraction time on human dentine permeability. Arch Oral Biol, v. 21, n. 10, p. 599-603, 1976.

20. PASHLEY, E. L.; LIWEN, T. S.; PASHLEY, D. H. Sterilization of human teeth: its effect on permeabillity and bond strength. Am J Dent, v. 6, n. 4, p. 189-191, Aug. 1993.

21. SETTEMBRINI, L.; GULTZ, J. P.; SCHERER, W.; KAIM, J. A single-component bonding system microleakage study. Gen Dent, v. 45, n. 4, p. 341-343, July 1997.

22. STRAWN, S. E.; WHITE, J. M.; MARSHALL Jr., G. W. et al. Spectroscopic changes in human dentine exposed to various storage solutions - short term. J Dent, v. 24, n. 6, p. 417-423, Nov. 1996.

23. TERUYA, J. I. Influência de diferentes técnicas de inserção na microinfiltração marginal de restaurações de resina composta em cavidade de classe II. São Paulo, 1994. 106 p. Dissertação (Mestrado em Materiais Dentários) - Faculdade de Odontologia, Universidade de São Paulo.

24. VIANNA, R.; BASTOS, E.; PRIMO, L. Restaurações proximais em molares decíduos: qual o melhor material? In: GONÇALVES, E. A. N.; FELLER, C. Atualização na Clínica Odontológica. São Paulo : Artes Médicas, 1998. Cap. 18. p. 443-464.

25. WU, W.; COBB, E.; DERMANN, K.; RUPP, N. W. Detecting margin leakage of dental composite restorations. J Biomed Mater Res, v. 17, n. 1, p. 37-43, Jan. 1983.

Recebido para publicação em 10/12/1999

Enviado para reformulação em 26/06/2000 Aceito para publicação em 05/12/2000 